\title{
Grammar: From Experience to Language
}

\author{
Zhongxin Dai \\ College of Foreign Languages, North China Electric Power University, Beijing, China \\ Jun Liu \\ College of Foreign Languages, North China Electric Power University, Beijing, China
}

\begin{abstract}
This paper attempts to address the nature of language grammar from the perspective of personal experience. Personal experience refers to whatever an individual, situated in a particular life status and equipped with certain innate mental and physical capacities, obtains in his interaction with the natural and socio-cultural surroundings, including various events that he has experienced, knowledge that he has acquired, feelings and emotions that he has gone through, cognitive abilities that he has developed, and whatever that makes him differ from what he was and that will contribute to later processes of his experience acquisition. Personal experience is an individual's lived experience, which enables him to understand the world, to understand what language means, and to behave appropriately in social and verbal communication. With the lived personal experience, an individual can come up with an appropriate communicative notion in a certain communicative context. The communicative notion is the consciousness or intentionality that the individual intends to communicate with the person to be addressed in the context. The communicative notion has its own meaningful structure, from which language grammar originates and develops in the process of serving the purpose of the communicative notion. Grammar is the realization of the communicative notion through the expression of language.
\end{abstract}

Index Terms - grammar, personal experience, language, communicative notion

\section{INTRODUCTION}

Just as Tomasello (2009, p.1) observed, psychologists have often waited for linguists to tell them what language is so that they can go on to study its comprehension, processing, and acquisition. But unfortunately much of the theoretical framework and vocabulary of modern linguistic theories relies on the categories and terminology of traditional Western linguistics. Linguists have failed to provide a ready and adequate theoretical framework for the study of language. Psychologists have to go out of their way to study the evolution, development, acquisition, comprehension and processing of language, on behalf of linguists. Language is in the speaker, used between people and situated while being acquired. Therefore, the focus of research on language itself is bound to fail in revealing the nature of language. Halliday (2002) also experienced the constraining rather than enabling walls of disciplines in the past century. He made some reference to the fact that in the mid-century many linguists sturdily proclaimed the independence and autonomy of the discipline of linguistics. Linguists should study language, he said, but they are not in a position to determine what questions should be asked about the domain of language. Halliday also pointed out that linguists always have been located and located themselves within some broader context. "[A]t any given 'moment' of space-time, there are likely to be only a few predominant motifs by which the context of linguistic scholarship is defined."

The paradigm of research has currently shifted to the cognitive approach to language. This approach to language aims to address the complex relationship between social language, personal experience, and mental cognition. Dai (2004) formulated the theory of personal experience (PE) in his doctoral dissertation The Formation and Development of Verbal Communicative Competence in a Foreign Language: A Perspective of the Personal Experience Theory. PE refers to whatever an individual, situated with a particular life status and equipped with certain innate mental and physical faculties, obtains by means of interaction with the natural and socio-cultural surroundings, including various events that he has experienced, knowledge that he has acquired, feelings and emotions that he has gone through, cognitive abilities that he has developed, and whatever that makes him differ from what he was and that will contribute to later processes of his experience acquisition. Language experience is part of the personal experience, which is always situated in social interaction and serves the purpose of communication. If language is regarded as a tool for communication, then grammar is the operational mechanism of the language tool. A tool is used for what it is intended to be used. Discussion of the language tool entails the discussion of the process of communication. Language originates and develops in the presence of the speaker's communicative notion, that is, part of the speaker's consciousness or intentionality that he attempts to communicate with the person that he is addressing. This way of reasoning enables us to see the nature of grammar. Like the development of any physical tool for labor, the development of the language tool has been targeted towards the fulfillment of its function and the ornamental or decorative design. A glass is container made of glass for drinking. It originates and develops from humans' need for drinking a liquid. It is humans' need that compels them to invent a container. No matter what material it is made up of, clay, stone, ceramic, metal or glass, the basic and fundamental function of a glass is "its usefulness as a container for drinking". The design of the glass is its 
ornamental feature. Likewise, the language is a tool for humans' communication. The function of language is to meet humans' need to express their communicative notion. It is not important whether it is realized by gestures or vocal sounds. Different languages are like the different materials that a container is made up of. The basic and fundamental function of different languages is the same. Some redundant rules of language are developed for the sake of perfection, formality or agreement of the rules. Functionally speaking, these rules are redundant and useless in the expression of the communicative notion. They are there only to demonstrate the beauty and human cognition of the language form. For instance, English language has the grammatical category of plurality of nouns. We say "Don't put all your eggs in one basket." The plural form "eggs" should be used. But when we say "There are plenty more fish in the sea", we use "fish". "Fish" is considered as an exception to the rule of plurality. Chinese and some other languages do not have plural forms for common nouns, but that does not affect the use of the languages. In the above English sentences, "all" and "more" already indicate the plural, so the plural forms are redundant. If words symbolize abstract concepts, then the agreement of the verb with the subject is also redundant.

Ontologically speaking, humans are beings in nature and society. According to Max-Neef (1991), fundamental human needs stem from the condition of being human. They are constant through all human cultures and across historical time periods. Personal experience is the experience that an individual obtains while he is being in a natural and socio-cultural situation. All psychological, emotional, cognitive and retrospective activities are done through and upon the personal experience. Since social communication is a fundamental human need and like all other fundamental human needs it is the same through all human cultures and across historical time periods, the PE and language-related phenomena are bound to be universal through all human languages, with grammar as part of it.

This article attempts to address the nature of the grammar of language from the PE perspective. We try to argue (1) the communicative notion comes from PE; and (2) grammar originates from and serves the purpose of the communicative notion. Hence the title, grammar comes from experience via the communicative notion.

\section{PE: The ORIGIN OF THE COMMUNICATIVE Notion}

We have all heard Aesop's fable story The Fox and the Crow. The Fox saw the Crow fly off with a piece of cheese in its beak and settle on a branch of a tree. The Fox must have understood what that meant. How did he know? He understood it from his lived experience. He co-lived with the Crow and the Crow revealed her being to the Fox, and hence he knew how to deal and communicate with the Crow. Personal experience is an individual's lived experience. Dell Hymes's (1972) communicative competence includes knowing when, where and how to speak, what to speak about, with whom, and so forth. Speakers need knowledge not only of what is grammatically possible but also of what is appropriate and typically done. If we ask further questions about the origin of the knowledge, again we come to the lived experience of the speakers. A person comes up with different communicative notions when he meets different people, say, his boss, his wife and his children. When he is with his different children, he also has different conversations. He may discuss the problem in the work with his boss, his wife's birthday party with his wife, and the English proficiency test with one of the children. Language communication starts from the lived experience of a particular individual.

At any given moment of space-time, an individual is experiencing a particular and specific situation in life. Dewey (1938) discussed two principles of experience: the principle of interaction and the principle of continuity. The first principle means that experience is both the process and the result of one's interaction with the external world; the second principle is to say that experience is continuous process; one's lived experience has a driving force, affecting the manner of his interaction with whatever he will experience. At any given moment of space-time, we are at this cross-point of continuum and interaction of experience. We are confined to our lived experience, which affects, in one way or another, the way we feel and interact with our surroundings.

Dai (2011) elucidated, from a perspective of the PE theory, the cognition and relevance involved in verbal communication. The process of verbal communication, based upon PE, consists of two sub-processes that are heterogeneous in nature: the sub-process of the formation of the communicative notion, which is non-linguistic in nature, and the sub-process of verbal expression of the communicative notion, which is linguistic in nature. The speaker's personal experience enables him to turn an external context into an internal context, and develop a communicative notion and an utterance image, which is then verbally expressed. Liu $(1988,2001)$ views the process of verbal communication as a "double-layer" process, with one layer being non-linguistic and the other linguistic. Some researchers of cognitive linguistics (e.g. Langacker, 1987, 1991, 2008; Evans 2009) also adopt this "double-layer" view towards the process of language understanding and production. Liu (2001) argues that the reason why the same utterance is subject to different understandings is that different people draw different inferences when they convert the literal meaning of the utterance into contextual meaning and speaker's intention. Then why are people liable to draw different inferences from the same utterance? Liu provide two reasons: the meaning in an utterance consists of elements different in nature; and different people have different ways of cognition.

According to Sperber and Wilson (2001), communication is an ostensive-inferential process. In the communication process, the speaker should make his intended information explicit, and the listener associates the information with the context and infers the speaker's intention. Then how it comes that different people draw different inferences? How can we account for the following talk between husband and wife who have just quarreled? 
Husband: Be careful on your way to work.

Wife: Don't worry. I won't kill myself in a car accident.

Dai (2011) pointed out that the interpretation of practical problems such as this in real verbal communication entails the explanation of the mechanisms involved in verbal communication from the perspective of personal experience. Verbal communication begins with the speaker's intention, which is brought about by the intentionality or consciousness that occurs to him in the presence of the listener. That is the communicative notion. The communicative notion arises out of the speaker's need for social communication. The study of verbal communication process should not just focus on the verbal communication process alone. The formation and development of the communicative notion should also be included, and viewed as the starting point of the process of verbal communication. Otherwise, the utterance during the process of verbal communication would seem to emerge out of thin air.

This theory on the verbal communication process interpreted from the perspective of the PE theory attempts to analyze daily routine verbal communication and to provide a clearer, more human and more practical framework for the analysis. Langacker (2013, p.1) argued that his cognitive grammar is intuitively natural, psychologically plausible and empirically viable. Verbal communication interpreted from the PE theory meets these standards of advantage, and more. It comes to the origin of grammar, universal or particular. It attempts to explore why grammar is intuitively natural, psychologically plausible and empirically viable.

\section{GRAMMAR AND THE COMMUNICATIVE Notion}

The speaker's personal experience gives rise to the communication notion, and his verbal expression is directed and targeted to the communicative notion. This expressing-and-expressed relation can be vividly illustrated in Vygotsky's (1986, p.251) description of the relation between thought and speech:

Thought, unlike speech, does not consist of separate units. When I wish to communicate the thought that today I saw a barefoot boy in a blue shirt running down the street, I do not see every item separately: the boy, the shirt, its blue color, his running, the absence of shoes. I conceive of all this in one thought, but put it into separate words. Speaker often takes several minutes to disclose one thought. In his mind the whole thought is present at once, but in speech it has to be developed successively. A thought may be compared to a cloud shedding a shower of words. Precisely because thought does not have its automatic counterpart in words, the transition from thought to word leads through meaning. In our speech, there is always the hidden thought, the subtext. Because a direct transition from thought to word is impossible, there have always been laments about the inexpressibility of thought.

Vygotsky implied that what is to be communicated is separated from the language with which the speaker to communicate what is to be communicated. What is to be communicated is the communicative notion, the "thought" in Vogosky's terminology. This communicative notion comes from the speaker's personal experience. The speaker experienced a barefoot boy in a blue shirt running down the street. This image of the communicative notion has its own structure: the "boy" is the agent; the "barefoot" and the "blue shirt" are the characteristics of the boy; the action of "be running" is what the boy, the agent, is doing; and "in the street" is place where the boy did the running. If the speaker is asked to tell more about what he experienced, he might be able to add more information to what he said. Other people who did not have the experience are unable to do so.

This is what linguists like Chomsky (1965) called "universal grammar". Universal grammar is the structure of the communicative notion. With this universal grammar in mind, humans create and develop their particular languages. The development of human language and children's language acquisition entail the perfection of articulation and of the complicated structure of the expression that matches the complicated structure of the communicative notion, and what is more important, as a tool for communication, language is conventional. This is quite typical of children's language acquisition and of adults' language production in real communication.

In Vygotsky's example, an adult may say "The boy." In his mind, he has the communicative notion of "Today I saw a barefoot boy in a blue shirt running down the street." This communicative notion can be realized in speech in various ways. For instance, "I saw a boy running in the street today. He had no shoes, and wore a blue shirt." Or "I saw a boy in the street. He was barefoot, and had a blue shirt. He was running down the street." No matter how the speech is worded, the meaning is the same because the communicative notion is the same. Grammatically correct sentences are the result of a long-term endeavor to perfect language expression towards the convention of language.

Dai (2004) imagined a situation of a black dog running after a hare. He said that both humans and other animals can experience the situation. The experience of the situation does not require language of any kind. On a certain occasion of social communication, the men in the making through social labor are compelled to communicate the communicative notion of a black dog running after a hare. It was then and only then that they had something to say to each other. This communicative notion is the prerequisite for the creation and development of human language. Human experience is the source of his conception of the "black", "dog", "running after" and "hare". The concepts of "black", "dog", "running after" and "hare" represent the elements of the notion and are realized by the phonological words. No matter how a language socially or collectively conventionalizes the structure of the notion, it should be capable of expressing the communicative notion. Hence Dai concluded that the structure of the natural world and the social one through the perception and conception of humans, the structure of the communicative notion that is formed from the experience within the natural and social world and the structure of language expression are identical and should be viewed in this 
logical and sequential order.

It is also quite plausible to conclude that the concepts of "black", "dog", "running after" and "hare" in the communicative notion might eventually be separated from the whole notion through various communicative notions formed on various occasions. On one occasion, the black dog is running after a hare; on another, a hare is grazing on the grass. There is no denying the fact that different languages have developed different conventions not only in the domain of grammar, but in the domain of conception of words. In one culture, the notion of "black dog" may be one compound concept, that is, it may be contained in one word; in another, it may be two. In one language, "black" is placed before "dog"; in another, after "dog". What Chomksy (1965) claimed as "universal grammar" is actually the "grammar" of the perceived world, and the "grammar" of the personal experience and the "grammar" of the communicative notion, whether it be called "grammar" or "structure" or "logic". A sentence can be divided into a noun phrase and a verb phase not because language is naturally and logically so, but because humans perceive the world in this way, and language is conventionalized accordingly.

A Chinese scholar and linguist named Lin Yutang (1982, p.1) observed in as early as the 1930s in the Preface "The Science of Expression" of his Kaiming English Grammar that "[i]n everything we say, there are always (1) something to say, and (2) the way of saying it. We shall call the former the notion, the latter the expression of these notions. We must understand that grammar has no meaning for us except to teach us about these notions and the ways of expressing them." Hence he $(1982$, p.7) advocated that to study grammar is "to look at grammar from the inside, and start from the idea we wish to express to its expression, from inner meaning to outer form." What Lin proposed is exactly what we have discussed here. In learning English as a foreign language, the learner should first learn to understand the notion that a certain grammatical structure expresses, and then attempt to imagine a situation where the speaker forms a communicative notion that needs to be expressed by the structure. For instance, the subjunctive structure "If ... had done something, ... would have done something." Just imagine that it rained yesterday and you did not have the picnic. It was a pity. Put yourself in the situation and imagine that you are saying to someone "If it hadn't rained yesterday, we would have had the picnic." You will have to direct your thought back to "the rain yesterday" and "the picnic that you did not have."

\section{CONCLUSION}

Cognitive approaches to language emphasize the effect of human experience of the world, the unique way humans perceive and conceptualize that experience, and how these are in turn reflected in the structure of language itself. A tool of any kind is never developed for the sake of the tool per se. Dai and Liu (2012) discussed the origin of tools and languages. They argued that "the crucial point in the creation process of tools and language is the meditation shifting towards a third party for the needs satisfaction. The shifting of meditation is the recognition of the usefulness of another thing to fill the void that emerges when the need arises." Language is a tool for communication, more precisely, a tool for the expression of the communicative notion. The creation of the language tool as a system for the expression of the communicative notion involves perception, emotions, categorization, conceptualization, and reasoning. All these cognitive abilities interact with the communicative notion. The nature of language is its function as a symbolic tool, i.e. the symbolic sign pointing to the communicative notion to be expressed. Theoretically speaking, language does not rely on the sound medium. Any signs (for instance, gestures) can be used as a symbolic tool that serves to fill the void between the communicative notion and the linguistic expression addressed to the listener.

According to Croft and Cruse (2004, p.1), there are three major hypotheses guiding the cognitive approach to language: language is not an autonomous cognitive faculty; grammar is conceptualization; and knowledge of language emerges from language use. These three hypotheses are not in the same picture. The first one is a claim against Chomsky's theory, repeatedly heard in theoretical works of cognitive linguistics; the second one is talking about the cognition involved in the creation and comprehension of human languages; and the third one is about the acquisition of language through the social interaction of verbal communication. The PE theory discussed in this paper has elucidated the phylogenetic and ontogenetic origins and development of language and grammar as a part of the language system. Viewed from the PE perspective, human language is simply easy to understand and analyze. Just imagine the two pictures: the mental and psychological picture of the communicative notion (non-linguistic in nature) and the symbolic picture of linguistic expressions (linguistic in nature, but also mental and psychological). Both Saussure (1983) and Chomsky $(1957 ; 1965)$ realized that language exists in the speaker's mind. Saussure chose "langue" and Chomsky "linguistic competence" as the target of study, but both of them miss the real target. The truth of language is in the history of the being of language, and this being of language should be viewed as co-being of language and humans in the real situation of human beings working with the language in social communication.

\section{REFERENCES}

[1] Chomsky, N. (1957). Syntactic structures. The Hague: Mouton \& Co. N.V., Publishers.

[2] Chomsky, N.(1965). Aspects of the Theory of Syntax. Boston, Mass.: MIT Press.

[3] Croft, W. \& Cruse, D. A. (2004). Cognitive Linguistics. Cambridge: Cambridge University Press.

[4] Dai, Zhongxin, \& Liu, Jun. (2012). The origin of language: A perspective of meditation shifting towards needs satisfaction. Global Journal of Human Social Science, 12(4), 1-5. 
[5] Dai, Zhongxin. (2004). The Formation and Development of Verbal Communicative Competence in a Foreign Language: A Perspective of the Personal Experience Theory (Doctoral Dissertation). Beijing: Beijing Normal University.

[6] Dai, Zhongxin. (2011). Cognitive relevance involved in verbal communication: A perspective of the personal experience theory. Theory and Practice in Language Studies, 1(8), 975-979. Doi: 10.4304/tpls.1.8.975979.

[7] Dewey, J. (1938). Experience \& Education. New York: Simon \& Schuster Inc.

[8] Evans, V. (2009). How Words Mean: Lexical Concepts, Cognitive Models and Meaning Construction. Oxford/New York: Oxford University Press.

[9] Halliday, M. A. K. (2002). Introduction: A personal perspective. In Jonathan J. Webster (ed.), On Grammar, pp. 1-12, New York: Continuum.

[10] Hymes, D. H. (1972). On communicative competence. In J. B. Pride, and J. Holmes (eds.), Sociolinguistics, pp. 269-93, Harmondsworth: Penguin.

[11] Langacker, R. W. (1987). Fundamentals of Cognitive Grammar: Volume I Theoretical Prerequisites. Stanford: Sanford University Press.

[12] Langacker, R. W. (1991). Fundamentals of Cognitive Grammar: Volume II Theoretical Prerequisites. Stanford: Sanford University Press.

[13] Langacker, R. W. (2008). Cognitive Grammar: A Basic Introduction. Oxford: Oxford University Press.

[14] Langacker, Ronald W. (2013). Essentials of Cognitive Grammar. Oxford/New York: Oxford University Press.

[15] Lin, Yutang. (1982). Kaiming English Grammar. Beijing: Foreign Language Teaching and Research Press. (Originally published by Kaiming Shudian in 1933.)

[16] Liu, Huanhui. (1988). Verbal Communication. Nanchang: Jiangxi Education Press.

[17] Liu, Huanhui. (2001). The Secret of Speech and Intention: Exploration of the Semantic Labyrinth of Utterances. Beijing: China Social Sciences Press.

[18] Max-Neef, Manfred A. (1991). Human Scale Development: Conception, Application and Further Reflections. New York/London: The Apex Press.

[19] Saussure, F. de. (1983). Course in General Linguistics. Edited by Charles Bally and Albert Sechehaye with the collaboration of Albert Riedlinger. Translated by Roy Harris. London: Gerald Duckworth \& Co. Ltd. (Original work published in 1916).

[20] Sperber, D \& Wilson, D. (2001). Relevance: Communication and Cognition ( ${ }^{\text {nd }}$ edn.). Beijing: Foreign Language Teaching and Research Press.

[21] Tomasello, M. (2009).Some surprises for psychologists. In M. Tomasello (ed.), The New Psychology of Language: Cognitive and Functional Approaches to Language Structure, pp. 1-14, New Jersey: Lawrence Erlbaum Associates, Inc.

[22] Vygotsky, L. S. (1986). Thought and Language (newly revised and edited by Alex Kozulin). Cambridge, Mass. Massachusetts Institute of Technology Press.

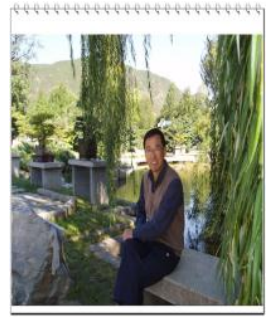

Zhongxin Dai was born in Hebei Province, China, in 1959. He received his B.A. in 1986, M.A. in 1989 in English language and literature, and PhD in 2004 in Education, all from Beijing Normal (Teachers) University. He was a visiting scholar at Peking University from 1995 to 1996, and at Exeter University from 1999 to 2000. He is currently a professor in the School of Foreign Languages, North China Electric Power University, Beijing, China. His research interests include second language acquisition, English learning and teaching, and cognitive linguistics.

Jun Liu is an associate professor of English at the School of Foreign Languages, North China Electric Power University. Her area of research is foreign language teaching and learning. 